\title{
Clinical and Histo-Pathological Evaluation of Fat Grafting in Chronic Leg Ulcers
}

\author{
AYMAN FARHAT, M.D.*; OSAMA EL-SHAHAT, M.D.*; ISMAEIL Al-KHWAGA, M.D.** and \\ MOHAMED IBRAHEM, M.B.B.Ch.* \\ The Departments of Plastic Surgery* and General Surgery**, Faculty of Medicine, Al-Azhar University
}

\begin{abstract}
Background: The application of autologous lipo-transfer (fat grafting) in reconstructive surgery becomes more popular now as an evidence for the regenerative effect of fat. In our study we used the autologous lipo-transfer for treatment of chronic ulcers at leg.

Methods: Twenty patients with chronic leg ulcers included in our study, ten of them injected with autologous fat (group A), while the other ten patients were on the usual dressings for chronic ulcers (group B), the duration of our study was for six months with patient age in both group range from 1550 years with mean age 35.3 years for injected group and 34.1 for the conservative group.
\end{abstract}

Results: Eight of ten chronic leg ulcers (80 percent) that injected with autologus fat healed completely within average of 8 weeks. With only 2 complicated, one complicated by infection while the other with resorption of fat. Only five patients at the conservative group healed within three months.

Conclusion: We described in our study a simple technique to improve wound healing in chronic leg ulcers with a background of vascular diseases, diabetic or traumatic accidents where other interventional options to achieve wound healing have failed.

Key Words: Fat grafting - Chronic leg ulcers.

\section{INTRODUCTION}

Chronic ulcers on the lower limb constitute particularly challenging situation with a high risk of morbidity for the patient.

Autologous fat transfer has been used to fill defects of soft tissues caused by trauma, deep burns or surgery. Adipose tissue has been shown to be an accessible source rich in mesenchymal stem cells (MSCs). These present an innovative therapeutic opportunity in many clinical fields including plastic and reconstructive surgery, especially for their ability to enhance mechanisms of repair in many tissues [1].
The major advantages of autologous fat grafting are the minimally invasive operation, the short hospital stay, the ease of harvest and implantation, low donor site morbidity, availability of fat and being autologous in nature, easy to learn and practice and minimal complications in competent hands [2].

Although autologous fat grafting has been shown to improve the quality and regenerative potential of chronically scarred tissues and burn scars, the effect on wound healing of chronic wounds and early-stage pressure ulcers has only been reported on sporadically. This study described a simple and useful technique of autologous lipotransfer to improve wound healing in chronic leg ulcers with a background of venous diseases, diabetic or in a traumatic manner.

A chronic skin ulcer (CSU) is defined as a wound lesion that lasts more than four weeks without remarkable healing tendency as a frequently recurrent wound that usually arrested in one of healing stages (usually the inflammatory one) [3].

So, in this study we evaluate the effect of fat grafting on healing of chronic leg ulcer.

\section{PATIENTS AND METHODS}

This prospective, non-randomized clinical study included twenty patients (13 male and 7 female) presented with chronic leg ulcers admitted to AlAzhar University Hospital and Banha Teaching Hospital between October 2016 and May 2017. Patients were fully informed about the procedure. Written consents were obtained. Patients were divided into 2 groups. Ten cases ( 7 male and 3 female) injected with autologus fat (group A) with mean age 35.3 years and another ten cases were on usual dressing for chronic ulcers (group B) (6 male and 4 female) with mean age 34.1. 
Inclusion and exclusion criteria:

Inclusion criteria:

- Patients with chronic leg ulcers.

- Aged 15-50 years.

- Size up to $4 \mathrm{~cm}^{2}$.

- It included both sexes.

- Non ischemic, non-infected limbs.

Exclusion criteria:

- Infected ulcers.

- Patients receiving drugs that delay healing (steroids, chemotherapy...).

- Uncontrolled DM.

- Ischemic ulcers.

- Patients refuse photography of their limbs.

- Patients suffered from cardio-pulmonary diseases.

- Malignant ulcers.

Surgical technique and autologous lipo-transfer method:

According to standard operating protocol, patients with group (A) underwent General or local anesthesia with intra venous sedation. Under complete aseptic conditions, the ulcer and the donor site were prepared using Betadine ${ }^{\circledR}$ surgical scrub (containing $7.5 \%$ povidone-iodine). Photographic documentation and wound swab was taken and sent for culture of organisms.

\section{Autologous lipotransfer:}

Preparation of the standard tumescent solution (sodium chloride) was performed. To each $500 \mathrm{ml}$ of $0.9 \%$ saline solution, one ampoule of adrenaline $1 \mathrm{mg} / \mathrm{ml}$ and $12.5 \mathrm{ml}$ of lidocaine hydrochloride $2 \%$ were added. After the preliminary incision of the skin with a scalpel blade ${ }^{\circ} 11$, we made infiltration of the donor areas, at the level of deep subcutaneous tissue, using a blunt cannula (tumescent infiltration cannula) filled with the particular anesthetic solution and allowed to take effect for a few minutes. Infiltration provides good hemostasis and adequate operative and peri-operative analgesic action. The fat was then harvested with a liposuction $3 \mathrm{~mm}$ cannula (merceds cannula) on a $50 \mathrm{cc}$ syringe from same the same incision we made infiltration before. The Available donor sites were lower abdomen (especially in obese) $(n=7)$, thigh and buttocks (in thin muscular patients) $(\mathrm{n}=1)$ and from flanks $(n=2)$. The harvesting sites were selected keeping in mind enhancement of the patient's contour. The aspirated fat was transmitted to $10 \mathrm{ml}$ syringes then centrifuged at (3000 rpm) for 3 minutes (Coleman technique) to concentrate the fat particles and separate them from fluids and debris which are then discarded [4].

After the centrifugation we obtain, inside the syringe, three distinct layers. The top layer, which is the less dense one, is made of oil derived from the breakdown of fat cells; in the intermediate portion we find adipocytes and stromal vascular tissue; in the lower level, the denser one, we find fluid. As the only layer that we need for the therapeutic purpose is the intermediate one, the oily layer is absorbed by tissue strips, and the lower layer is removed by removing the cap from the syringe, in this way permitting the spontaneous drainage of the aqueous portion, the purified fat was then transferred to $3 \mathrm{ml}$ Luer lock syringes to be ready for injection.

The $3 \mathrm{ml}$ Luer-Lock syringe with fat injection blunt cannula $(0.7 \mathrm{ml})$ attached to its nozzle was used.

The fat was infiltrated with a threading technique using minimal pressure (Fig. 1), in the direction from the surrounding areas (approximately $1 \mathrm{~cm}$ from the wound edge) toward the ulcer, or parallel to the wound edges, to avoid contaminating the surrounding tissues because of possible infection present in the wound. Fat was infiltrated retrogradely into the tunnels left by the cannula with each slow push. This technique seems to allow better fat graft survival, to increase rooting and to minimize the possibility of forming cavities filled with triglycerides. The amount of injected fat at each passage is minimized to avoid irregularities and clusters, which are eventually deleted with digital manipulation after procedure. Injection is performed with retrograde technique leaving a very small space between the injected tissue lines. The access incisions in the donor areas are sutured with vicryl 3/0.

Punch biopsy has been taken from the ulcer (intra-operative) including the bed, the ulcer edge and the skin surrounding the ulcer for a histopathological specimen.

The treated area was covered with gauze dressing for 3-5 days and the patients were instructed not to apply pressure or friction to the recipient sites to limit the displacement of fat infiltration. External pressure with elastic compressive dressing was applied to the donor sites to minimize postoperative edema and to prevent hematoma formation.

\section{Post-operative care:}

- Patients were managed in the Plastic and Reconstructive Surgery Department for several 
hours and further evaluation of post-operative outcome was performed in the outpatient clinic. All patients received antibiotics for 7 days, antiedematous and analgesic drugs.

- The first look for the recipient site was on the third day post operative day. Regarding to the donor site, the first dressing was performed on the fifth post-operative day. Sutures were removed 7 -
10 days postoperative. Results were assessed by clinical examinationand post-operative photographs three days and one week postoperatively then at 1, 3 and 6 months postoperatively (Figs. 2,3).

- The postoperative outcome was evaluated according to the therapeutic response which was determined according to size of ulcer if it decreased or not.
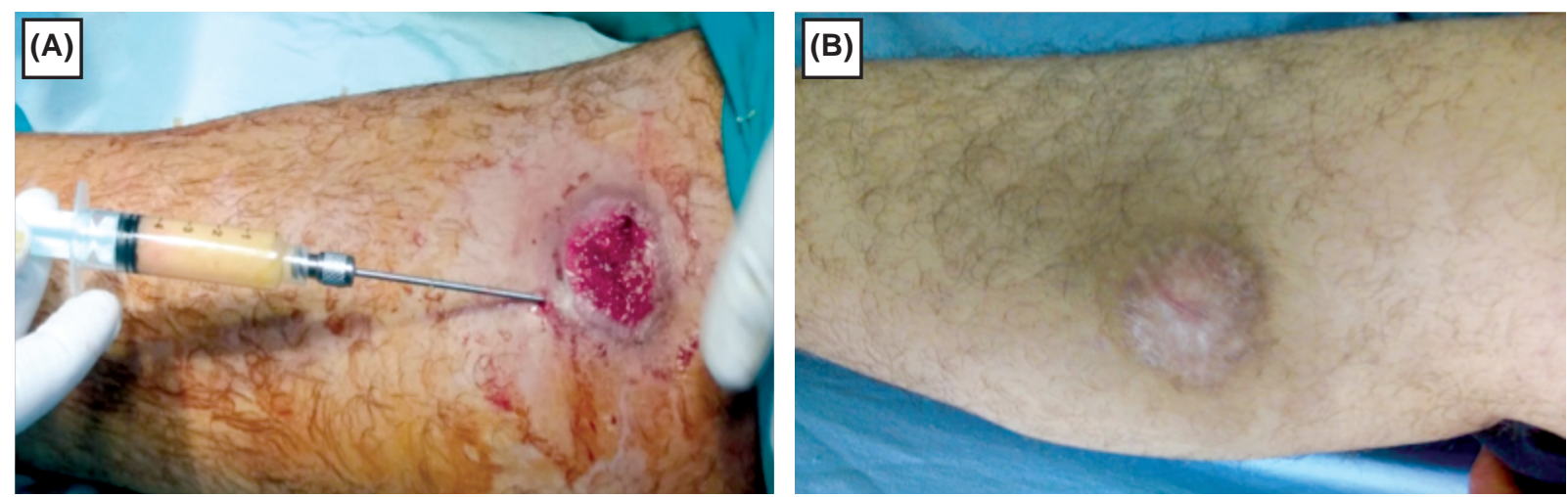

Fig. (1): Male patient 38 years old with post traumatic chronic ulcer with 3 months duration at right leg, he has been injected with fat at ulcer edges and its bed (A), effect of injection appeared only after 2 weeks with reduction size of ulcer and begin to heal, till complete healing of the ulcer reaching normally as normal skin after 45 days (B).

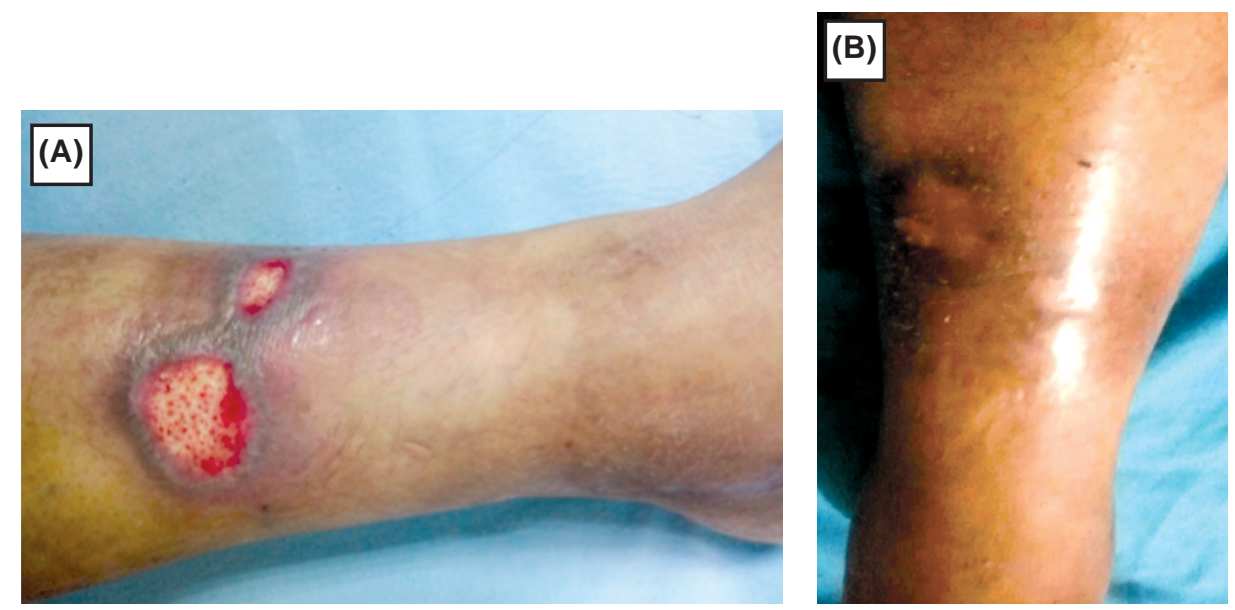

Fig. (2): Male patient 44 years old with chronic venous ulcers $(n=2)$ of 4 months duration and was no progression on usual dressings the patient used (A). The ulcers were injected with fat with slight reduction at first 2 weeks till complete healing and closing of both achieved within 2 months $(\mathrm{B})$.
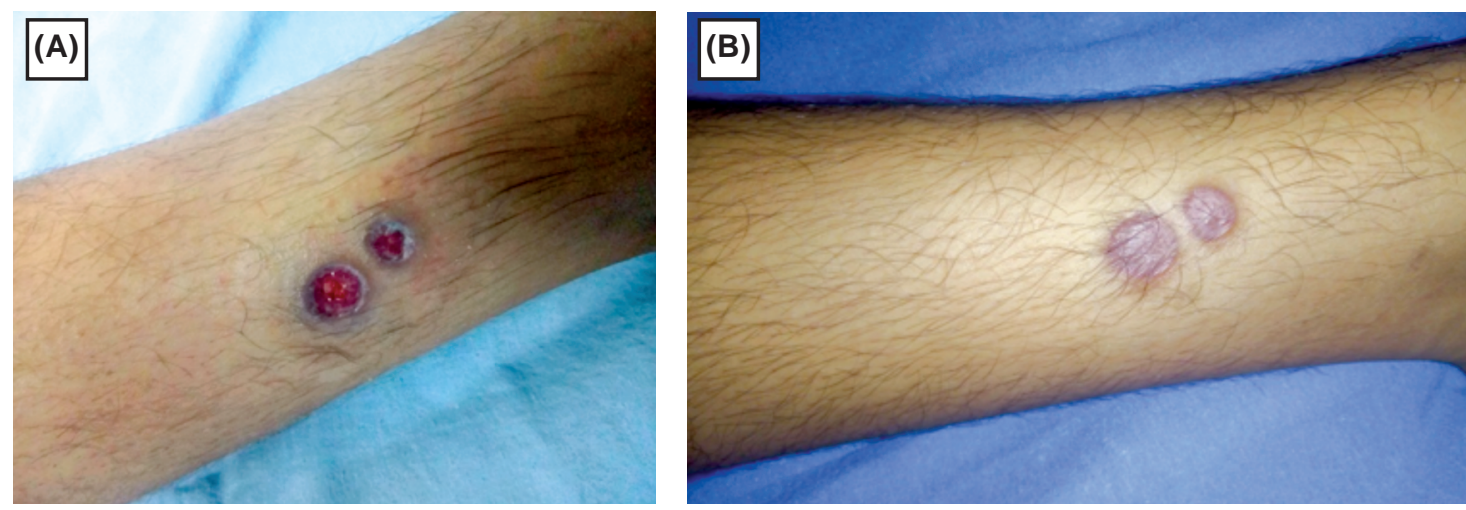

Fig. (3): Male patient 18 years old with 2 traumatic ulcers with 2 months duration resistant for closure (A), after fat injection they took only 10 days for closing the wound then complete healing achieved within one month (B). 


\section{RESULTS}

Our study consisted of 20 patients in two groups: Injected group $(n=10)$ and control (conservative) group $(\mathrm{n}=10)$ with a mean age $34.7 \pm 11.62$ with a range of 35 and median of 31 , with a minimum of 15 and maximum of 50 .

Age:

The two groups were matched in age through using Mann-Whitney Test. The results of Mann Whitney are shown in Table (1).

The results shown above indicate that there are non-significant differences between the two groups in age. Z-value was 0.265 (n.s).

The study showed that $20 \%$ were in the age range $15-20,45 \%$ in the age range $25-40$, and $35 \%$ in the age range $40-50$.

\section{Gender:}

The two groups consisted of male and female participants: The injected group (injected by fat) consisted of 7 males and 3 females whereas control group consisted of 6 males and 4 females. As the percent of male in our study was $65 \%$ while female was $35 \%$. The two groups were matched in gender using chi square test. The results are showed in Table (2).

\section{Type of ulcers:}

We performed our study on 3 types of ulcers which are traumatic, venous and diabetic. The percentage of patients with venous was $30 \%, 15 \%$ for diabetic, and $55 \%$ for traumatic ulcers. The two groups were matched in type of ulcer using chi square test. The results are showed in Table (3).

\section{Ulcer duration:}

The two groups were matched in duration of ulcer using chi square test. The least duration of an ulcer was 1 month while the longest duration was an ulcer of 8 months. It has been noticed that there is inversely proportion relation between the duration and rate of healing since that the healing is faster when duration (chronicity) is less. The results are showed in Table (4).

\section{Reduction size of ulcer:}

The reduction size of ulcer was compared between the two groups across time through comparing the number of patients in every week for five weeks after excluding the patients who have not changed. The results are shown in Table (5). The study showed that in injected group most of size reduction happened in first week $(\mathrm{n}=4)$ with around $50 \%, 2^{\text {nd }}$ week $(\mathrm{n}=2)$ with $25 \%$ and third week $(\mathrm{n}=2)$ with $25 \%$.

\section{Complete healing:}

The complete healing of ulcer was compared between the two groups (Tables 6,7) across time through comparing the number of patients in every week for five weeks after excluding the patients who have not changed.

The previous tables shows the effect of fat grafting by enhancing the healing of ulcers and shorten the duration too in comparison to the conservative group.

\section{Donor site:}

Lower abdomen was the donor site in most cases ( 7 cases) due to easy accessibility and abundant fat, thigh was selected in (1 case) and flanks in ( 2 cases) as shown in Table (8).

\section{Volume of fat injected:}

As shown in Table (9) the results were recorded as volume of fat injection in the ulcers. The amount of fat increases according to size of ulcer.

\section{Complications:}

As regards the complications one case showed fat resorption and another one shows infection as shown in Table (10) with no any significant problem or complication at other cases.

Table (1): Mann Whitney results for matching the two groups.

\begin{tabular}{ccccccccc}
\hline Group & Mean & SD & Median & Min. & Max. & Z & Sig. \\
\hline Age & Injected & 35.30 & 12.15 & 37 & 15 & 50 & 0.265 & n.s \\
& $\begin{array}{l}\text { Conser- } \\
\text { vative }\end{array}$ & 34.10 & 11.47 & 36 & 15 & 50 & & \\
\hline
\end{tabular}

Table (2): Chi square results for matching the two groups (gender).

\begin{tabular}{llcccc}
\hline & Group & Male & Female & Chi & Sig. \\
\hline \multirow{2}{*}{ Gender } & Injected & 7 & 3 & 0.220 & n.s \\
& Conservative & 6 & 4 & & \\
\hline
\end{tabular}

Table (3): Mann Whitney results for matching the two groups (type of ulcer).

\begin{tabular}{llccccc}
\hline & Group & Venous & Traumatic & Diabetics & Chi & Sig. \\
\hline Gender & Injected & 2 & 6 & 2 & 1.091 & n.s \\
& Conservative & 4 & 5 & 1 & & \\
\hline
\end{tabular}


Table (4): Mann Whitney results for matching the two groups (duration).

\begin{tabular}{lccccccc}
\hline Group & $\begin{array}{c}1-2 \\
\text { months }\end{array}$ & $\begin{array}{c}2-3 \\
\text { months }\end{array}$ & $\begin{array}{c}3-6 \\
\text { months }\end{array}$ & $\begin{array}{c}\text { Mor 6 } \\
\text { months }\end{array}$ & Chi & Sig. \\
\hline Duration Injected & 1 & 2 & 6 & 1 & 2.260 & n.s \\
Conservative & 2 & 7 & 0 & 1 & & \\
\hline
\end{tabular}

Table (5): Shows number of ulcers decrease in size according to weeks.

\begin{tabular}{lcc}
\hline Weeks & Injected group & Conservative group \\
\hline First week & 4 & 0 \\
Second week & 3 & 2 \\
Third week & 2 & 4 \\
Fourth week & 1 & 0 \\
\hline
\end{tabular}

Table (6): Complete healing of injected group.

\begin{tabular}{lcl}
\hline Days & $\begin{array}{c}\text { Number of patients with } \\
\text { complete healing }\end{array}$ & $\%$ \\
\hline $0-15$ days & 1 & 12.5 \\
15-30 days & 2 & 25 \\
$30-45$ days & 3 & 37.5 \\
$45-60$ days & 2 & 25 \\
\hline
\end{tabular}

Table (7): Complete healing of conservative group.

\begin{tabular}{lcl}
\hline Months & $\begin{array}{c}\text { Number of patients with } \\
\text { complete healing }\end{array}$ & $\%$ \\
\hline $1-2$ months & 2 & 20 \\
$2-3$ months & 3 & 30 \\
$3-4$ months & 0 & 0 \\
$4-6$ months & 0 & 0 \\
No complete healing & 5 & 50 \\
\hline
\end{tabular}

Table (8): No. and percent of donor site.

\begin{tabular}{lcl}
\hline Donor site & Number of patients & $\%$ \\
\hline Abdomen & 7 & 70 \\
Anterior high & 0 & 0 \\
Lateral high & 1 & 10 \\
Flanks & 2 & 20 \\
\hline
\end{tabular}

Table (9): Volume of fat injection.

\begin{tabular}{lcc}
\hline Volume of fat injected & Number of patients & $\%$ \\
\hline $2-4 \mathrm{cc}$ & 3 & 30 \\
$4-8 \mathrm{cc}$ & 6 & 60 \\
$8-12 \mathrm{cc}$ & 1 & 10 \\
\hline
\end{tabular}

Table (10): Complications in the studied cases.

\begin{tabular}{lcc}
\hline Complications & Number of patients & $\%$ \\
\hline Infection & 1 & 10 \\
Fat resorption & 1 & 10 \\
No complication & 8 & 80 \\
\hline
\end{tabular}

\section{DISCUSSION}

Chronic ulcers on the lower limb constitute a particularly challenging situation with a high risk of morbidity for the patient, often associated with recurrent surgical debridement and, eventually amputations in a compromised vascularized environment our study shows the enormous effect of autologous lipo-transfer on wound healing as relatively easy-to-perform, cost-effective, and well tolerated procedure.

Marino et al., described in his study the effect of lipo- injection on chronic leg ulcers of 20 patients suffering from peripheral arterial diseases. Ten of them injected with fat founding that there is a reduction in both diameter and depth of all ulcers injected and six of them were completely healed. He was depending on the celution technique, which is a solution of ADRC (adipose derived regenerating cells) isolated by a specific celution system $[\mathbf{5 , 6}]$ for 2 hours from liposuctioned adipose fat, depending on the regeneration system of ARDC, while in our study we used the coleman technique depending on centrifuging ( $3000 \mathrm{rpm}$ in 3 minutes) and gave good result in regeneration as 8 of 10 cases we injected with fat were completely healed. The underlying causes of ulcers in our study was venous, Diabetic or traumatic with no arterial (ischemic) ulcers.

Caviggioli reported in his study (a case study) that he did 2 sessions of autologus-lipo transfer with the Coleman technique for treatment of post traumatic leg ulcer with about $7.5 \mathrm{~cm}$ in diameter which healed completely [7]. In our study we used only one session of fat grafting in traumatic ulcers with good results as maximum diameter was $4 \mathrm{~cm}$, which may be attributed to the small ulcers size compared to caviggioli study.

Cervelli et al., added platelet-rich plasma to enhance the wound healing properties of fat grafting. They used platelet gel from a small volume of blood ( 9 to $18 \mathrm{ml}$ ) followed by injection of fat graft obtained with the Coleman technique. Their results demonstrated the efficacy of combining these two treatments but here in our study we did not add prp so we cannot judge the effect of its adding, but fat injection alone was enough for complete healing [8].

Mohan described in his study (a case study) that he used not only autologus lipo transfer (2 sessions) in treatment of post-radiation ulcer, but also skin grafting in addition. The 10 years history chronic leg ulcer was completely healed after 11 months from the previous procedures ( 2 sessions 
of fat grafting and skin grafting) [9]. In our study we did not operate on radiation ulcers or depending on skin grafting. But in further studies we hope to see the effect of that combination especially in different types of ulcers.

Tilman et al., showed in his study that was for 26 patients with chronic leg and foot ulcers, 22 of them were completely healed within a mean of $68.0 \pm 33.0$ days and reduction of wound size by 50 percent was achieved after an average of 4 weeks and that by making debridement firstly for ulcers, injected them with fat and application of vac therapy for enhancing angiogenesis and further healing [10].

Sophie Bartsich showed in his study (a case study) that he used debridement a with fat and skin grafting for treatment of patient suffering from chronic sickle cell ulcer and hepatic (hepatitis c). The ulcer completely healed within 6 weeks, so combination can be used also to overcome some systemic problems [11]. Our study was depending only on fat injection and free of any systemic diseases except for controlled diabetes.

Histo-pathological specimen has been taken from the ulcer and its edges showing signs of healing as proliferation of fibroblast and increasing angioangesis but it is important to keep in mind that cancerous tissue can be a cause for non-healing wounds so in our study we ruled out ulcers of suspicious cancerous tissue and fortunately it was no any cancerous ulcers.

\section{Conclusion:}

The use of fat grafting in therapeutic processes related to Reconstructive Surgery is considered to be now a good option for enhancing healing as cheap and easy to perform. Our study showed the regenerative effect of adipose tissue in treatment of chronic leg ulcers. The excellent results shown in recent studies have only served to confirm the good outcomes described in our study. We hope in further studies to illustrate the effect of adding of combining skin grafting, prp into lipo-injection fat grafting on another different types of ulcers.

\section{REFERENCES}

1- Gronthos S., Zannettino A.C.W., Hay S.J., Shi S., Graves S.E., Kortesidis A. and Simmons P.J.: Molecular and cellular characterisation of highly purified stromal stem cells derived from human bone marrow. Journal of Cell Science, 116: 1827-35, 2003.

2- Kaminer M.S. and Omura N.E.: Autologous fat transplantation. Arch. Dermatol., 137 (6): 812-814, 2001.

3- Christopher E.A., Jeffrey E.J., Steinberg, Schwartz, Ali A. and Kara C.: Clinical Approach to Wounds: Debridement and Wound Bed Preparation Including the Use of Dressings and Wound-Healing Adjuvants. Plastic Reconstructive Surgery, 117 (Suppl.): 72S, 2006.

4- Coleman S.R.: Structural fat grafting: More than permanent filler. Plastic and Reconstructive Surgery, 118: 108-120, 2006.

5- Lin K., Matsubara Y., Masuda Y., et al.: Characterization of adipose tissue-derived cells isolated with the Celution system. Cytotherapy, 10: 417, 2008.

6- Marino G., Moraci M., Armenia E., et al.: Therapy with autologous adipose-derived regenerative cells for the care of chronic ulcer of lower limbs in patients with peripheral arterial disease. J. Surg. Res., 185: 36-44, 2013.

7- F. Caviggioli, D. Forcellini, V. Vinci, G. Cornegliani, F. Klinger and M. Klinger: "Employment of needles: A different technique for fat placement," Plastic and Reconstructive Surgery, Vol. 130: pp. 373e-374e, 2012.

8- Cervelli V., Gentile P. and Grimaldi M.: Regenerative surgery: Use of fat grafting combined with platelet-rich plasma for chronic lower-extremity ulcers. Aesthetic Plast Surg., 33: 340-345, 2009.

9- Mohan A. and Singh S.: Journal of wound care, Vol. 26, No 5 May 2017.

10- Tilman Stasch, Julius Hoehne, Tuan Huynh Randy De Baerdemaeker, Siegfried Grandel and Christian Herold: Volume 136, Number 6 Débridement and Autologous Lipotransfer by the American Society of Plastic Surgeons, 2015.

11- Bartsich S. and Norman Morrison WOUNDS: 24 (3): 51, Volume 24 - Issue 3 - March 2012. 